\title{
Methoxycarbazolyl-disubstituted dibenzofuranes as holes- and electrons- transporting hosts for phosphorescent and TADF-based OLEDs
}

\author{
Audrius Bucinskas ${ }^{\mathrm{a}}$, Oleksandr Bezvikonnyi ${ }^{\mathrm{a}}$, Dalius Gudeika ${ }^{\mathrm{a}, \mathrm{b}}$, Dmytro Volyniuk ${ }^{\mathrm{a}}$, \\ Juozas V. Grazulevicius ${ }^{\mathrm{a}, *}$ \\ a Department of Polymer Chemistry and Technology, Kaunas University of Technology, Radvilenu Pl. 19, LT-50254, Kaunas, Lithuania \\ ${ }^{\mathrm{b}}$ Institute of Solid State Physics, University of Latvia, Kengaraga St. 8, Riga, Latvia
}

A R T I C L E I N F O

\section{Keywords:}

Methoxycabazole

Dibenzofuran

OLED

Host

\begin{abstract}
A B S T R A C T
In the search of universal host materials for organic light emitting diodes a new series of bipolar host materials containing methoxy-substituted carbazoles as the electron-donating and dibenzofuran as an electron-accepting units were designed and synthesized. Different linking topologies and number of methoxy groups attached to carbazolyl moiety were used to understand the impact of the strength of the donor moiety on the thermal, optical, photophysical, electrochemical and electroluminescent properties. The synthesized compounds exhibited relatively high thermal stability with $5 \%$ weight loss temperatures exceeding $378^{\circ} \mathrm{C}$ and formed molecular glasses with high glass-transition temperatures ranging from 120 to $148{ }^{\circ} \mathrm{C}$. High triplet energy values of 2.86-2.96 eV were estimated for dilute THF solutions at 77K. Hole and electron drift mobilities estimated using time-of-flight technique in solid layers approached $10^{-4} \mathrm{~cm}^{2} \mathrm{~V}^{-1} \mathrm{~s}^{-1}$ at high electric fields exceeding $3.6 \times 10^{5} \mathrm{~V} \mathrm{~cm}^{-1}$. The synthesized methoxy-carbazole based compounds were tested as hosts in electrophosphorescent and TADF organic light-emitting diodes reaching luminance of $53000 \mathrm{~cd} \mathrm{~m}^{-2}$ and external quantum efficiency of $12.5 \%$, in the best case.
\end{abstract}

\section{Introduction}

As the first generation fluorescent OLEDs rely only on the singlet excitons (25\%), most of the energy (75\%) is lost through non-generated triplet excitons. This was a huge limitation for large scale applications. The employment of triplet excitons using host-guest system, typically, with transition metal complexes, in case of PHOLEDs, or noble-metalfree organic molecules, in case of TADF OLEDs, resulted the increase of the external quantum efficiency from 4 to $8 \%$ [1,2] up to $31 \%[3,4]$. To get effective light generation employment of triplet excitons is important, but not less important to avoid or diminish the quenching pathways. Therefore, one of the fundamental factors in the design of effective OLEDs is selection of a host for host-guest system. In principle, host materials should maintain the following requirements: high triplet energy, appropriate HOMO and LUMO levels, bipolar charge transporting properties, etc. [5]. Many host materials were reported for either phosphorescent or TADF OLEDs [3,6-11]. However, incomparably less research was done on the search for versatile host materials, which can be applied both for PHOLEDs and TADF OLEDs. Many effective OLEDs were designed using hole-transporting and host materials containing carbazole moiety as electron-donating unit [5,12]. In most cases, carbazole is selected due to high triplet energy, good thermal stability and useful optical and charge-transporting properties of carbazole derivatives [13]. Carbazole-disubstituted derivatives of dibenzofurane were previously reported [14]. It was suggested to use them as host materials for PHOLEDs. In this work, we modified carbazole moiety by introducing methoxy groups to C-2, C-3, C-6 and C-7 positions, seeking to improve the whole bunch of properties. Our first goal was to increase electrochemical stability of modified new host materials [15]. By linking methoxy groups to the most reactive positions of carbazole moiety (C-3 and C-6) we obtained compounds which showed reversible oxidation [16]. Secondly, due to enhancement of hydrogen bonding between oxygen atoms of methoxy groups and hydrogen atoms of other molecules efficient packing is achieved in films resulting in high thermal stability, high glass transition and effective charge transport $[8,9,17,18]$. Thirdly, it is known that upon methoxy substitution, HOMO energies of the compounds are increased and ionization potential values are decreased resulting in more effective hole injection [19-21]. However, to our knowledge, no derivatives of methoxy-substituted carbazole capable of effectively transporting both

\footnotetext{
* Corresponding author.

E-mail address: juozas.grazulevicius@ktu.lt (J.V. Grazulevicius).
} 
holes and electrons were reported. Therefore, it is of interest to further study structure-properties relationship of methoxy-substituted carbazoles. In this study, three donor-acceptor-donor compounds were designed and synthesized as bipolar host materials containing electrondonating methoxycarbazole and electron-accepting dibenzofuran units as versatile host materials for both PHOLEDs and TADF-based OLEDs. Photophysical, thermal, electrochemical electrooptical and charge transporting properties of the compounds were investigated. Taking into account the results of investigations, the hosts were utilized in phosphorescent and TADF OLEDs discussing effects of substitution patterns on device performances.

\section{Results and discussion}

Our approach makes use of 2,8-dibromodibenzofuran [8] as precursor of an electron-accepting chromophore and methoxy-substituted carbazole as precursor of electron-accepting unit for the synthesis of DA-D systems. Electron-donating building blocks (2,7-dimethoxycarbazole, 3,6 dimethoxycarbazole and 2,3,6,7-tetramethoxycarbazole) were synthesized according to our previously reported procedures $[9,13]$. The target compounds F36DMCz, F27 DMCz and FTMCz were prepared by Ullmann-coupling reactions using nitrobenzene as solvent (Scheme 1). [8].

\section{Thermal properties}

The behaviour of F36DMCz, F27DMCz and FTMCz under heating was investigated by DSC and TGA under nitrogen atmosphere. The temperatures of thermal transitions are listed in Table 1. The first DSC heating scans confirmed that compounds F36DMCz, F27DMCz, FTMCz were obtained as crystalline materials with the melting point values of 256, 225 and $291{ }^{\circ} \mathrm{C}$, respectively. No transition peaks associated with crystallization peaks were observed during the DSC cooling scans, which indicated formation of glasses. In the second DSC heating scans, the compounds exhibited glass transitions with glass transition
Table 1

Thermal characteristics of F36DMCz, F27DMCz and FTMCz.

\begin{tabular}{llll}
\hline Compound & $\mathrm{T}_{\mathrm{m}},\left[{ }^{\circ} \mathrm{C}\right]^{\mathrm{a}}$ & $\mathrm{T}_{\mathrm{g}},\left[{ }^{\circ} \mathrm{C}\right]^{\mathrm{b}}$ (2nd heating scan) & $\mathrm{T}_{\mathrm{d}},\left[{ }^{\circ} \mathrm{C}\right]^{\mathrm{c}}$ \\
\hline F36DMCz & 256,275 & 121 & 424 \\
F27DMCz & 225 & 123 & 420 \\
FTMCz & 291 & 149 & 378
\end{tabular}

a $\mathrm{T}_{\mathrm{m}}$ is melting temperature at the scan rate of $10^{\circ} \mathrm{C} / \mathrm{min}, \mathrm{N}_{2}$ atmosphere.

b $\mathrm{T}_{\mathrm{g}}$ is glass-transition temperature.

c $\mathrm{T}_{\mathrm{d}}$ is the temperature of initial weigh loss at the scan rate of $20^{\circ} \mathrm{C} / \mathrm{min}, \mathrm{N}_{2}$ atmosphere.

temperatures $\left(\mathrm{T}_{g}\right)$ ranging from 121 to $149^{\circ} \mathrm{C}$. The $\mathrm{T}_{\mathrm{g}}$ value for FTMCz was found to be higher by $26-29^{\circ} \mathrm{C}$ in comparison to those of F27 DMCz and F36DMCz. This is likely due to the stronger intermolecular interactions, i.e. larger hydrogen-bonding network [14], in the sample of FTMCz which contains twice more methoxy groups in carbazole moiety than F36DMCz and F27DMCz. F36DMCz, showed crystallization in the second heating scan at $180^{\circ} \mathrm{C}$ followed by melting at $275^{\circ} \mathrm{C}$. Crystallization from the bulk yielded crystals with melting point higher by $19^{\circ} \mathrm{C}$ than that of crystals obtained by crystallization from solution (Fig. 1).

All the compounds demonstrated relatively high thermal stability. The temperatures of the onsets of weight loss $\left(\mathrm{T}_{\mathrm{d}}\right)$ were in the range of 378-424 ${ }^{\circ} \mathrm{C}$ (Fig. 1b). Compounds F36DMCz and F27 DMCz having four methoxy groups in their structures exhibited comparable $\mathrm{T}_{\mathrm{d}}$, whereas FTMCz derivative having eight methoxy groups demonstrated by $40^{\circ} \mathrm{C}$ lower $\mathrm{T}_{\mathrm{d}}$ values. The similar effect was observed in the previous work [17].

\section{Electrochemical and photoelectrical properties}

Electrochemical properties of F36DMCz, F27DMCz and FTMCz were evaluated by cyclic voltammetry (CV) at room temperature using dichloromethane solutions. The energy level data is listed in Table 2. The CV peaks of the solutions (Fig. 2a) revealed that F36DMCz and<smiles>Brc1ccc2oc3ccc(Br)cc3c2c1</smiles>
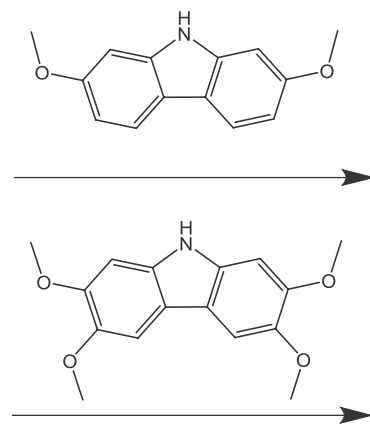

CONDITIONS: $210^{\circ} \mathrm{C}$, nitrobenzene, $\mathrm{Cu}$, base<smiles>COc1ccc2c(c1)c1cc(OC)ccc1n2-c1ccc2oc3ccc(-n4c5ccc(OC)cc5c5cc(OC)ccc54)cc3c2c1</smiles><smiles></smiles><smiles></smiles>

Scheme 1. Synthesis of F36DMCz, F27DMCz and FTMCz. 

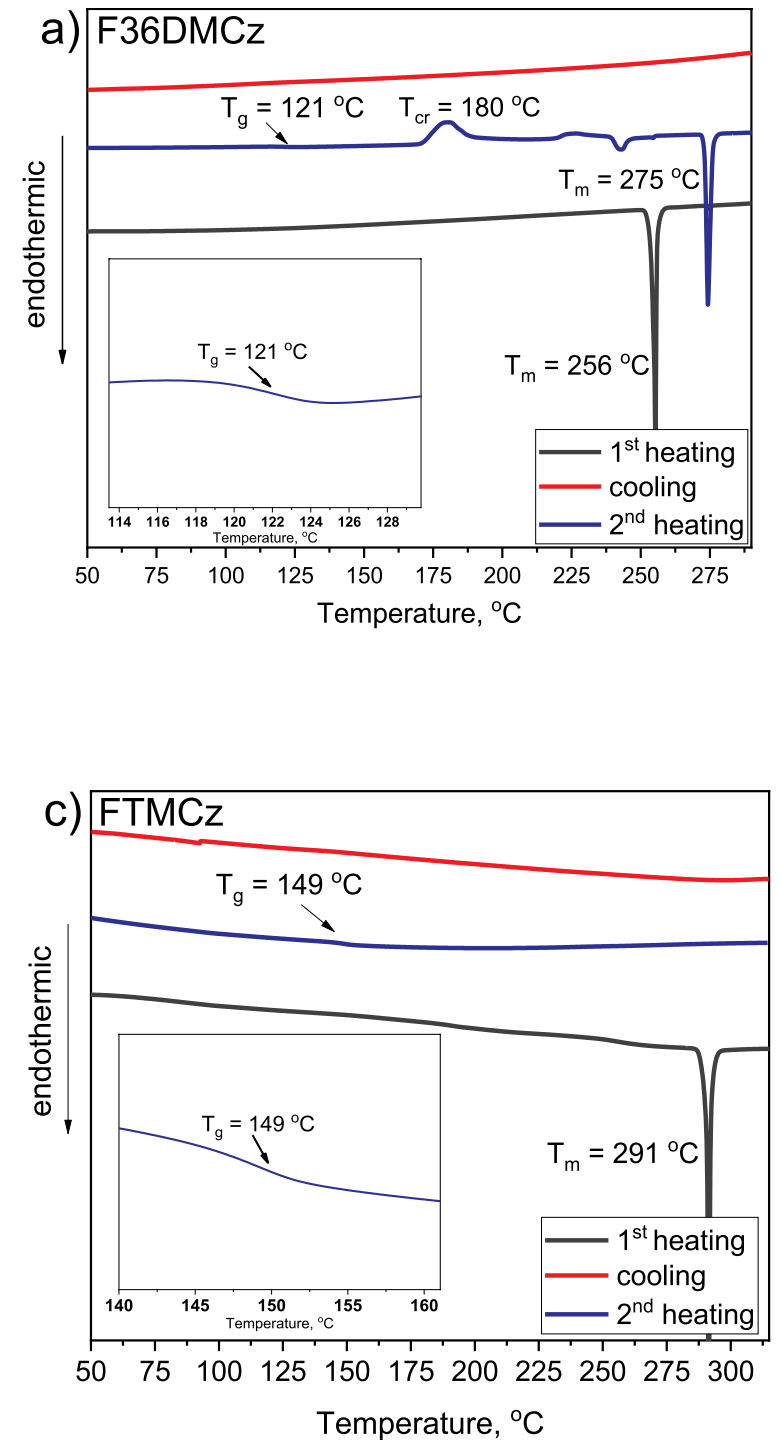
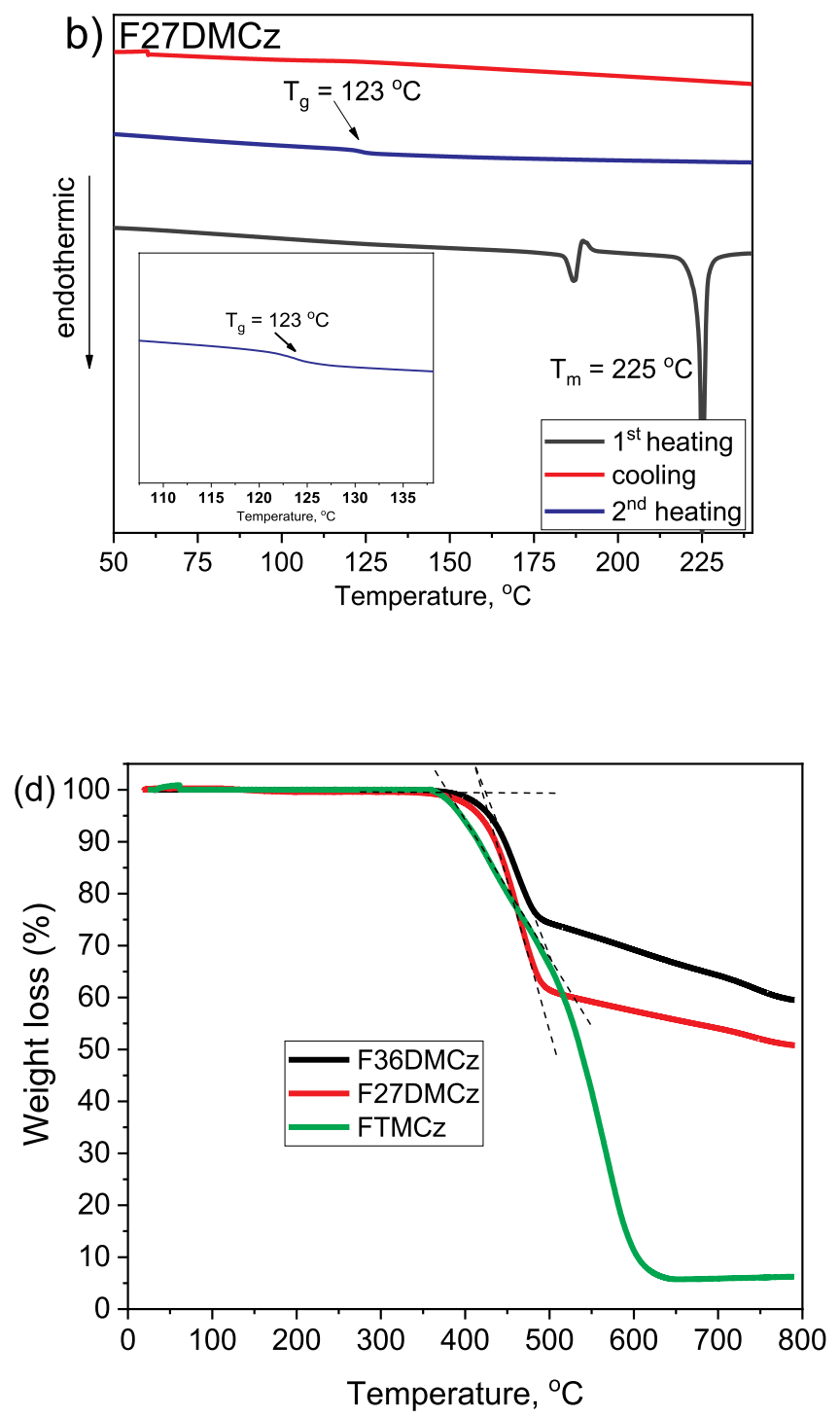

Fig. 1. DSC (a,b,c) thermograms and TGA (d) curves of F36DMCz, F27DMCz and FTMCz.

FTMCz exhibited single quasi-reversible oxidation (repeated scanning over 3 cycles) waves with an oxidative onset potentials of $0.44 \mathrm{eV}$ and $0.33 \mathrm{eV}$, respectively, while F27 DMCz showed irreversible oxidation with the onset potential value of $0.49 \mathrm{~V}$. Irreversible oxidation processes of F27 DMCz occurred by coupling of carbazole radical cations because of higher electron spin density at C-3 and C- 6 positions [22]. The oxidation peak values of the compounds were found in the range of $0.41-0.64 \mathrm{~V}$. No reduction waves were observed down to ca. $1.5 \mathrm{~V}$. The HOMO energy levels were determined using the relationship $\mathrm{E}_{\mathrm{HOMO}}$ $(\mathrm{eV})=1.4 * \mathrm{E}_{\text {onset,ox }}(\mathrm{V})+4.6$. ( $E_{\text {onset,ox }}$ is the onset of the first oxidation wave) [23]. The HOMO energy levels of F36DMCz, F27 DMCz and FTMCz derivatives were calculated to be of $-5.22,-5.29,-5.06 \mathrm{eV}$, respectively. The DFT-calculated (B3LYP/6-31G (d,p)) HOMO energy levels are in a good agreement with the experimentally estimated values (Table 2, Fig. 5). Compared to dimethoxycarbazole-based derivatives (F36DMCz, F27DMCz), tetramethoxycarbazole containing compound FTMCz showed higher HOMO energy value by $0.20-0.30 \mathrm{eV}$ proving the methoxy group effect, i.e. HOMO energy increase [9,24].

Ionization potential values of solid films of F36DMCz, F27DMCz, FTMCz were measured by photoelectron emission technique in air

Table 2

Electrochemical characteristics of F36DMCz, F27DMCz and FTMCz.

\begin{tabular}{|c|c|c|c|c|c|c|c|}
\hline Compound & $\mathrm{E}_{\text {onset, }} \mathrm{V}$ & $\mathrm{E}_{\text {ox,pa }} \mathrm{V}^{\mathrm{a}}$ & $\mathrm{E}_{\mathrm{ox}, \mathrm{pc}} \mathrm{V}^{\mathrm{a}}$ & $E_{1 / 2}, V$ & $\mathrm{HOMO}^{[\mathrm{b}]} / \mathrm{HOMO}_{\mathrm{DFT}}{ }^{\mathrm{d}}, \mathrm{eV}$ & $\mathrm{LUMO}_{\mathrm{DFT}}, \mathrm{eV}^{\mathrm{d}}$ & $\mathrm{IP}, \mathrm{eV}^{\mathrm{c}}$ \\
\hline F36DMCz & 0.44 & 0.55 & 0.45 & 0.50 & $-5.22 /-4.91$ & -1.34 & 5.35 \\
\hline F27 DMCz & 0.49 & 0.64 & 0.38 & 0.51 & $-5.29 /-4.97$ & -1.33 & 5.52 \\
\hline FTMCz & 0.33 & 0.41 & 0.34 & 0.37 & $-5.06 /-4.80$ & -1.27 & 5.35 \\
\hline
\end{tabular}

Each measurement was calibrated with ferrocene (Fc).

${ }^{\mathrm{a}} E_{o x, p a}, \mathrm{E}_{\mathrm{ox}, \mathrm{pc}}$-peak potentials corresponding to successive molecular oxidations.

b $E_{\text {Номо }}(\mathrm{eV})=-1.4 E_{\text {onset }}$ ox $(\mathrm{V})-4.6$.

c Ionization potentials of the solid samples estimated by photoelectron emission in air.

d Calculated values by the B3LYP/6-31G(d,p) method. 


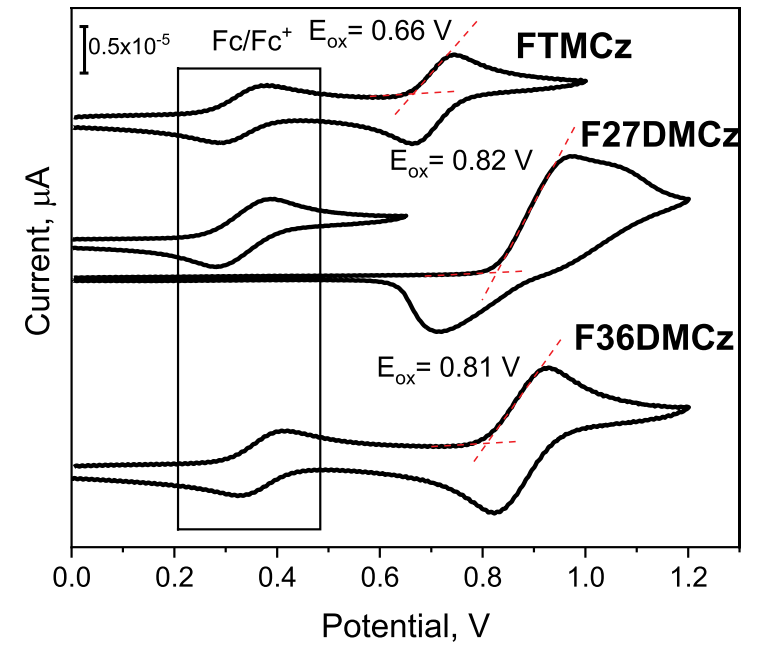

a)

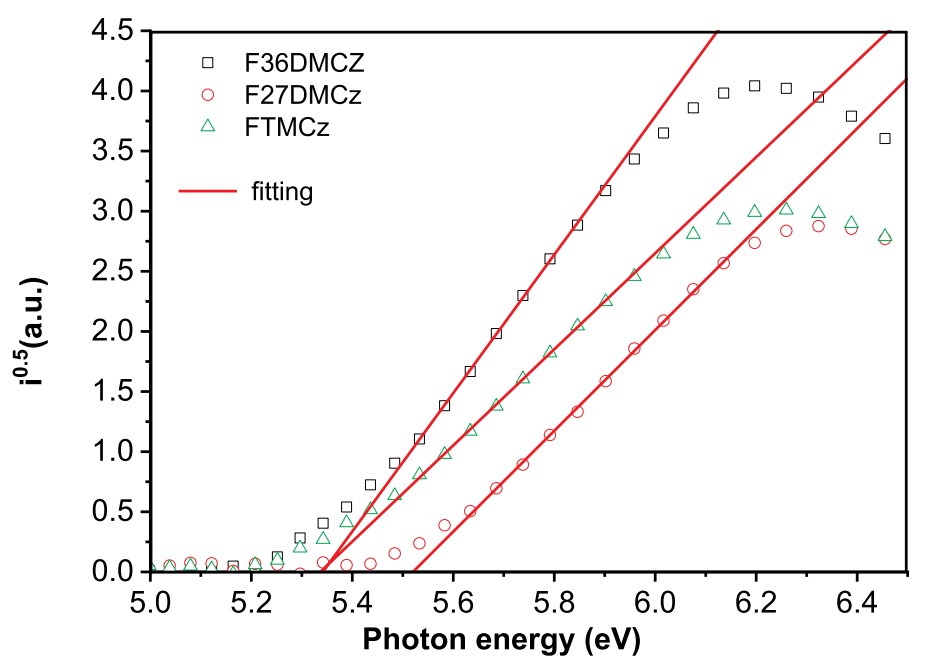

b)

Fig. 2. CV curves (a) and photoelectron emission spectra (b) of the layers of F36DMCz, F27DMCz, FTMCz.

(Fig. 2b). Being slightly higher than corresponding IP values measured by CV experiment but following the same trend, the IP values of the layers of the synthesized compounds ranged from 5.35 to $5.53 \mathrm{eV}$.

Effect of substitution pattern of carbazole moieties of the studied donor-acceptor compounds on their semiconducting properties was investigated conducting time of flight (ToF) measurements. Values of charge drift mobilities for the tested films of compounds were calculated taking transit times $\left(t_{\mathrm{tr}}\right)$ at cross point of tangent lines at different slopes of transient curves in log-log scales as it is shown in Fig. 3b. Such cross points were related to the transit times since their shifts to smaller times were clearly observed with increasing applied voltages. Due to the shapes of ToF curves, the films of F36DMCz were characterized by relatively low dispersive hole transport. Much more dispersive hole transport was detected for the films of F27 DMCz and FTMCz (Fig. S3). In addition, strongly dispersive electron transport was observed for the tested films. Relatively high errors of the determined values of transit times lead to errors in calculated electron mobilities (Fig. 3b, Fig. S3). Apparently because of the strongly dispersive electron transport, it was not possible to find transit times for the films F36DMCz and its electron mobilities were not estimated. Nevertheless, the values of hole mobility of compound F36DMCz exceeded $10^{-4} \mathrm{~cm}^{2} \mathrm{~V}^{-1} \mathrm{~s}^{-1}$ at high electric fields $\left(>3.6 \cdot 10^{5} \mathrm{Vcm}^{-1}\right)$. The values of hole mobility of compounds F27DMCz and FTMCz were several times lower at the same electric fields. These differences apparently related to the differently substituted methoxycarbazole units of the compounds. These differences may induce different intermolecular interactions and/or molecular packing. Electron mobilities of F27 DMCz and FTMCz were found in the similar range because of the same accepting moiety used in their molecular structures. Taking into account bipolar charge-transport, the studied compounds could be of interest as host materials for further testing in OLEDs where hole and electron transport is needed.

\section{Photophysical properties}

To study effect of differently substituted methoxycarbazoles on photophysical properties of the designed compounds, absorption and photoluminescent (PL) spectra were recorded of their toluene and THF solutions as well as of the films (Fig. 4a). Major photophysical characteristics are collected in Table 3. The well-structured low-energy absorption band (LEB) was observed for the solutions of F36DMCz in solutions. LEBs of the solutions of F27 DMCz and FTMCz were lessstructured but still observable. Despite donor-acceptor-donor structure of the compounds, their LEBs are very similar to LEBs of the corresponding methoxy-substituted carbazoles. They mostly relate to the local carbazole transitions as it was previously observed [13]. Presence of electron-accepting dibenzofuran unit in the molecular structures of investigated derivatives may result in formation low-energy absorption bands of charge transfer (CT) character. However they are practically
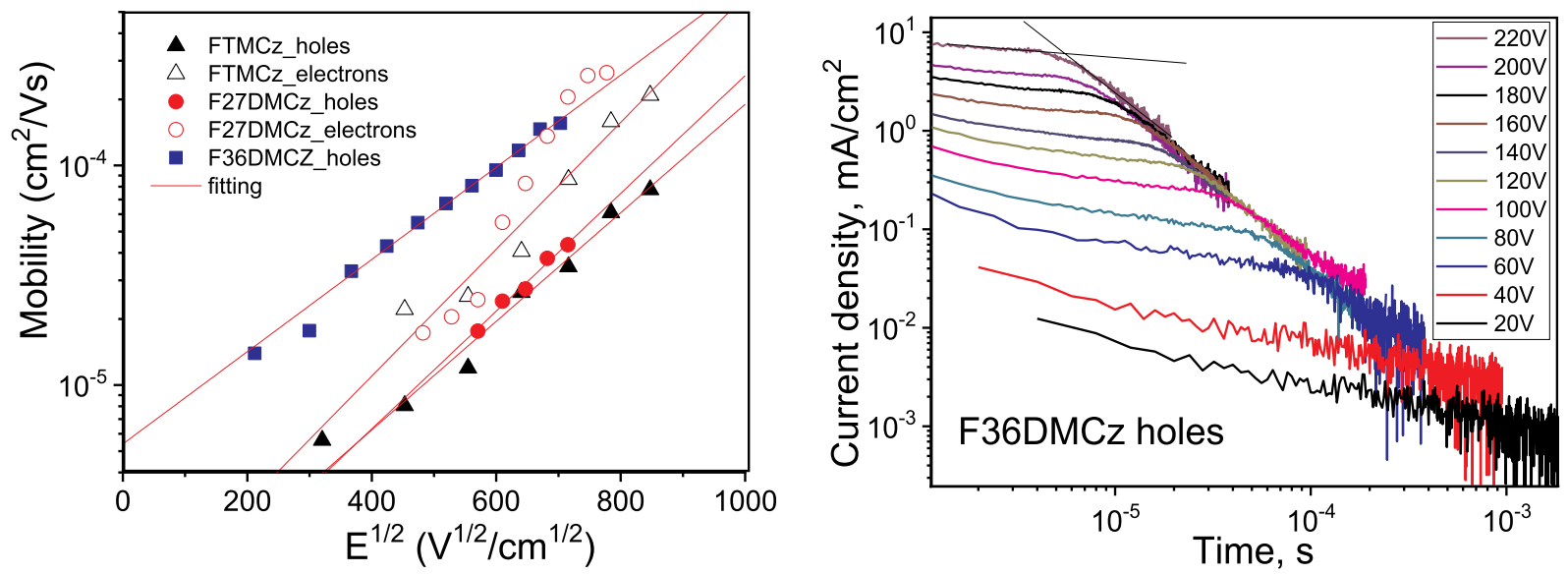

Fig. 3. Hole and electron mobilities versus electric field (a) and time-of-flight signals for holes for the film of F36DMCz at different voltages. 


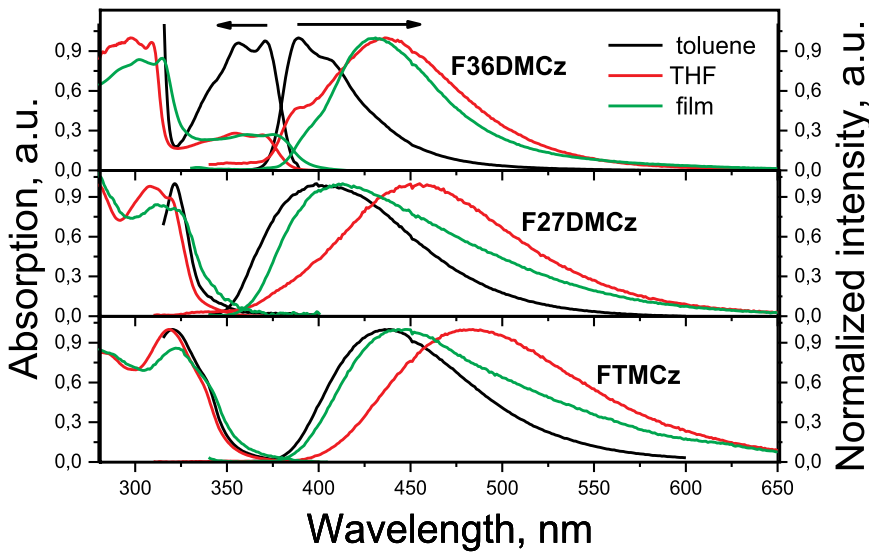

(a)

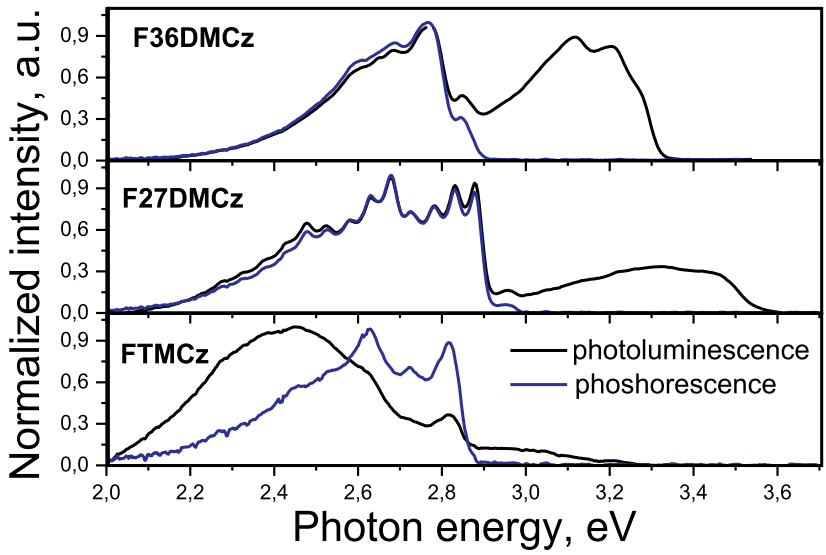

(b)

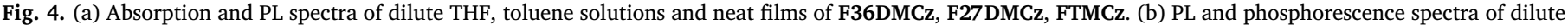
THF solutions recorded at $77 \mathrm{~K}$.

not detectable in absorption spectra of the solutions due to the insignificant interfragment $\pi$-conjugation (Fig. 4a). Red-shifts of absorption spectra of the films of the studied compounds are apparently related to formation of CT states between methoxycarbazoles and dibenzofurane units since interfragment interaction may be induced in solid films. The values of optical band gap estimated from the onset wavelengths of LEB of neat films and presented in Table 3.

The solution of F36DMCz in toluene demonstrated double peaked emission spectrum in contrast to those of other derivatives studied (Fig. 4a). The HOMO and LUMO distribution of F36DMCz shows that the dibenzofuran and donor moieties are not completely separated electronically (Fig. 5). PL band of toluene solution of F36DMCz can be mainly attributed to the radiative deactivation of the local excited state of methoxycarbazole chromophores because of its similarity to those of methoxycarbazoles [13]. In addition, the solution of F36DMCz in toluene exhibited emission of the intramolecular charge transfer (ICT) nature. ICT is manifested by the positive solvatochromic effect of dilute solutions. The emission peaks of the THF solution is bathochromically shifted in comparison to that of the solution in less polar toluene by ca. $50 \mathrm{~nm}$, while the change in the position of the corresponding absorption peaks is negligible. Such emission behavior of F36DMCz may be related to relatively weak donating ability of methoxycarbazole units attached at C-3 and C-6 positions of carbazole moiety, which determines weak CT emission of the solutions F36DMCz in the solvents of low polarity. In contrast, emission of toluene solutions of F27DMCz and FTMCz is characterized by broader spectra with a single maximum (Fig. 4a). CT character of F27DMCz and FTMCz emission is evidenced by red-shifts of emissions of the solutions in THF in comparison to those of the solutions in toluene. Thus, emission of the solutions of F27 DMCz and FTMCz is related to the transition from the LUMO of dibenzofuran moiety to the HOMO of di(tetra)metoxycarbazole unit dominates almost uncompetitively. This explanation is supported by spatial separation of HOMO and LUMO of F27DMCz and FTMCz. The most efficient charge transport is expected for the layers of F27DMCz due to the larger HOMO/LUMO distribution compared to that of $\mathbf{F} 36 \mathrm{DMCz}$ and FTMCz. Such consideration is in agreement with the largest Stokes shift observed for THF solution of F27DMCz. The different character of the radiative processes of derivatives can be explained by different donating abilities of di(tetra)metoxycarbazoles resulting in different distributions of the frontier molecular orbitals between these di(tetra) metoxycarbazole and dibenzofuran moieties (Fig. 5).

Fluorescence of the neat thin film of F36DMCz is very similar to ICT emission of dilute THF solution of the compound (Fig. 4a). In addition, high-energy shoulder is observed in PL spectrum of the film of F36DMCz. This shoulder is similar to emission of toluene solution peaked at 398. Solid-state emission of F36DMCz similarly to that of solutions results from recombination of the hybridized local and charge transfer (HLCT) state (mixed localized electronic $\left(\pi-\pi^{*}\right)$ and chargetransfer states) [25]. In contrast, fluorescence resulting from deactivation of only CT states was detected for the films of F27DMCz and FTMCz (Fig. 4a). In the cases of either HLCT or CT states, triplet excitons of investigated compounds may exhibit radiative decay processes as it was previously reported [18]. This presumption is in good agreement with the result of investigation of the effect of air on the emission of the compounds (Fig. S1). The increase of fluorescence intensity of both solid samples and dilute solutions was observed after deoxygenation. Maxima of PL spectra of the films of F27DMCz and FTMCz are observed close to the PL maxima of the corresponding toluene solutions indicating low polarity of F27DMCz and FTMCz [26].

Photoluminescence quantum yield (PLQY) values of the deoxygenated THF solutions and neat thin films of compounds are found to be identical for F27 DMCz and FTMCz (Table 3). In case of F36DMCz,

Table 3

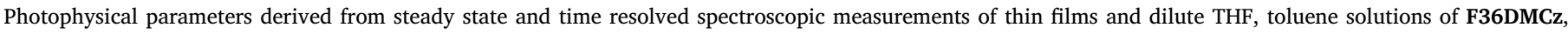
F27DMCz, FTMCz.

\begin{tabular}{|c|c|c|c|c|c|c|c|}
\hline Compound & $\lambda_{\mathrm{PL}}, \mathrm{nm}^{\mathrm{a}}$ & $E_{g}, e V$ & $\Phi_{\mathrm{THF}}^{\mathrm{b}}$ & $\Phi_{\text {film }}$ & $\mathrm{E}_{\mathrm{T} 1}{ }^{\mathrm{c}} / \mathrm{E}_{\mathrm{T} 1 \text { (theor) }}{ }^{\mathrm{d}} \mathrm{eV}$ & $\mathrm{E}_{\mathrm{S} 1}{ }^{\mathrm{c}} / \mathrm{E}_{\mathrm{S} 1 \text { (theor) }}{ }^{\mathrm{d}} \mathrm{eV}$ & $\Delta \mathrm{E}_{\mathrm{ST}}{ }^{\mathrm{c}} / \Delta \mathrm{E}_{\mathrm{ST}}$ (theor.), $\mathrm{eV}^{\mathrm{d}}$ \\
\hline F36DMCz & $393,431 / 389,404 / 389,437$ & 3.13 & 0.17 & 0.10 & $2.89 / 2.89$ & $3.32 / 3.07$ & $0.43 / 0.16$ \\
\hline F27DMCz & $410 / 398 / 452$ & 3.58 & 0.06 & 0.06 & $3.01 / 2.96$ & $3.55 / 3.18$ & $0.54 / 0.19$ \\
\hline FTMCz & $444 / 436 / 483$ & 3.47 & 0.11 & 0.11 & $2.86 / 2.86$ & $3.27 / 3.05$ & $0.41 / 0.17$ \\
\hline
\end{tabular}

\footnotetext{
a Wavelength of emission peak of neat thin film/dilute toluene solution/dilute THF solution.

b PLQY value of deoxygenated dilute THF solution.

c Estimated from emission spectra of dilute THF solutions recorded at 77K.

d Calculated values by DFT B3LYP/6-31(d, p) method.
} 


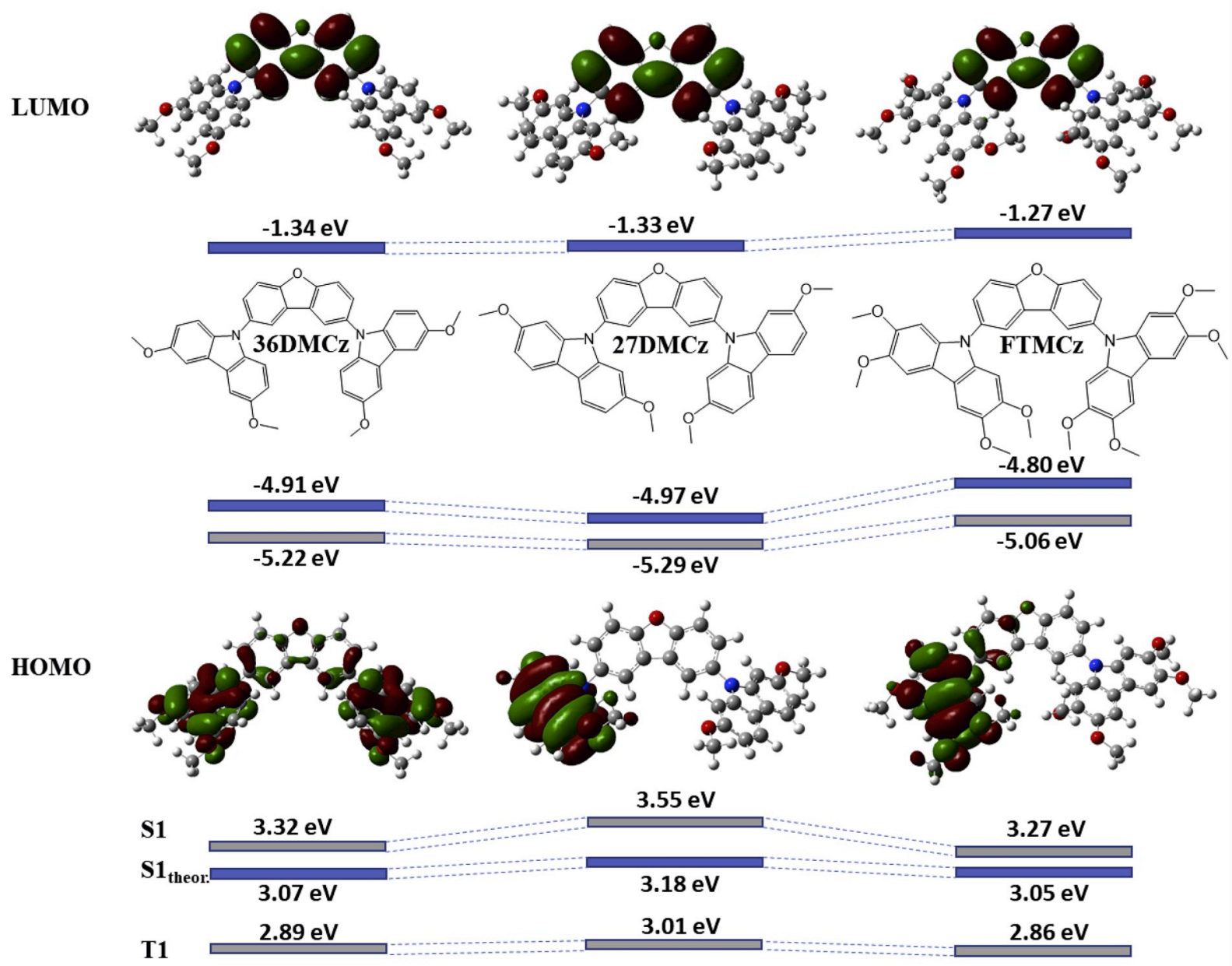

Fig. 5. Experimental (grey) and theoretical (blue) HOMO/LUMO levels and orbitals, $\mathrm{S}_{1}$ and $\mathrm{T}_{1}$ energies of F36DMCz, F27DMCz, FTMCz. (For interpretation of the references to colour in this figure legend, the reader is referred to the Web version of this article.)

PLQYs of 0.17 and 0.10 for THF solution and solid sample, respectively, are obtained. Low PLQY values of the solutions can apparently be explained by malleable flexible linkages between donor and acceptor units. As a result, free vibrations of methoxycarbazoles in solutions lead to emission quenching. Although these free vibrations are blocked in solid films, methoxy groups induce intermolecular interactions (e.g. hbonding [27]) leading to the aggregation induced emission quenching.

Host materials of OLEDs have to be characterized by higher triplet levels than energy levels of radiative states of emitter used. To examine triplet levels of studied derivatives, their phosphorescence and PL spectra of dilute THF solutions at $77 \mathrm{~K}$ were recorded (Fig. 4b). The shapes of phosphorescent spectra as well as the values of first triplet energy level of the compounds are in good agreement with those previously published compounds based on only methoxycarbazoles [18]. Thus, phosphorescence of THF solutions was completely related to recombination of triplet LE states of methoxycarbazoles. The influence of acceptor dibenzofuran on phosphorescence spectra of the compounds was not detectible. The values of first singlet and triplet energy levels, $E_{S 1}$ and $E_{T 1}$, were estimated from the onset of photon energies of the PL and phosphorescence bands, respectively (Table 3). The theoretically predicted $\mathrm{E}_{\mathrm{S} 1}$ and $\mathrm{E}_{\mathrm{T} 1}$ values of the compounds are in a good agreement with the experimental ones (Table 3, Fig. 5). Due to the high triplet levels of $2.86-2.96 \mathrm{eV}$, the investigated compounds can be recognized as promising host materials for OLEDs.

To demonstrate energy transfer from hosts F36DMCz, F27 DMCz or FTMCz to emitters, host-guest layers were fabricated using green TADF emitters DAcIPN [28] and DACT-II [29] as guests at $10 \mathrm{wt} \%$ doping concentration. PL spectra of these host-guest layers demonstrated single bands related to guest emissions and no bands/shoulders of hosts were recorded (Fig. S2a). Although the spectra of hosts are different, the fluorescence spectra of doped films are almost identical with wavelength of the emission peaks of ca. $555 \mathrm{~nm}$ for the films containing DAcIPN and $545 \mathrm{~nm}$ for ones with DACT-II indicating efficient hostguest interaction. PL decay curves of the doped films recorded at room temperature contained components in a $\mu$ s range which are attributed to TADF as it was proved elsewhere (Table S1, Fig. S2) [21,22]. These data show that the synthesized compounds can operate as hosts transferring electronic excitation energy to guest emitters without suppressing TADF.

\section{Device fabrication and characterization}

To investigate effect of methoxy substituents of carbazole moiety on the host efficiencies in TADF and phosphorescent OLEDs, comparable study was performed using available two TADF emitters (DAcIPN and DACT-II) and two phosphorescent emitters (tris[2-phenylpyridinato$\mathrm{C} 2, \mathrm{~N}]$ iridium(III) (Ir(ppy) ${ }_{3}$ and bis(1-phenylisoquinoline)(acetylacetonate)iridium(III) (Ir(piq) $)_{2}$ (acac)). HOMO/LUMO values of these emitters did not match well with HOMO/LUMO values of the studied hosts (Fig. 6). As a result, electroluminescent performances of the fabricated devices were lower than those of published OLEDs containing the same emitters [30-32]. Nevertheless, the comparable study provided evidence that compound F36DMCz demonstrated better host efficiencies relative to compounds F27 DMCz and FTMCz. 


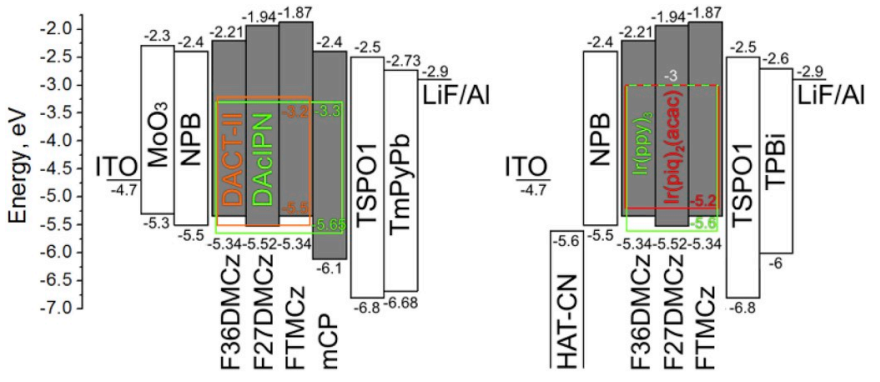

Fig. 6. Equilibrium energy diagram of OLEDs.

In order to estimate hosting properties of F36DMCz, F27DMCz and FTMCz, two series of OLEDs based on DAcIPN and DACT-II as TADF emissive species were fabricated. Additionally, the studied compounds were tested as hosts in two series of PhOLEDs using green and red emitters $\operatorname{Ir}(\mathrm{ppy})_{3}$ and $\operatorname{Ir}(\mathrm{piq})_{2}$ (acac). Device structures of the fabricated OLEDs as well as their major electroluminescent characteristics are collected in Table 4. The functional layers of the fabricated devices were selected for providing good charge injection, charge transport and recombination within light-emitting layers due to the appropriate energy levels of the materials as it is visualized in equilibrium energy diagram (Fig. 6). The layer of $\mathrm{MoO}_{3}$ or HAT-CN was used as hole injection layer. The layer of $N, N^{\prime}$-di(1-naphthyl)- $N, N^{\prime}$-diphenyl-(1,1'-biphenyl)-4,4'-diamine (NPB) was employed as hole transport layer. Diphenyl-4-triphenylsilylphenyl-phosphine oxide (TSPO1) was used as the hole blocking material. The layers of 1,3,5-tri(m-pyridin-3-ylphenyl)benzene (TmPyPb) and 2,2',2"-(1,3,5-benzinetriyl)-tris(1phenyl-1-H-benzimidazole) (TPBi) were employed as electron-transporting layers. The layer of LiF was used as the electron injecting layer. The anode and cathode were of ITO and aluminium, respectively.

The emission parameters of TADF OLEDs are shown in Figs. S3-9. EL spectra of all the devices contain single peak attributed to emission of the dopant. CIE coordinates and wavelengths of the spectral peaks (Fig. 7a, S3-9) correlate to a great extent with the performances of OLEDs based on other hosts reported earlier [21,22]. Good electronic excitation energy transfer from host to guest molecules is also evident from the absence of collateral peaks in EL spectra of OLEDs.
Having small differences in their molecular structures, hosts F36DMCz, F27 DMCz and FTMCz based on carbazolyl moiety with just different number and substitution pattern of methoxy groups induced essential differences in electroluminescence characteristics of the fabricated devices. Using TADF emitter DAcIPN in devices A, B, and C, the values of maximum EQE of $8.1>5>3.1 \%$ were obtained for these devices displaying the order of host efficiency F36DMCz > F27DMCz > FTMCz (Table 4, Fig. 7b). The same trend of host efficiency was obtained in case of the DACT-II-based devices E, $\mathrm{F}$, and $\mathrm{G}$ according to their maximum EQEs. The different device efficiencies are apparently related to the differences in properties (HOLO/ LUMO values, triplet values, structure/morphology of layers, etc.) of the hosts used. The obtained data put F27 DMCz in the middle between F36DMCz and FTMCz with respect of efficiency of TADF OLEDs (Table 4). Based on the fact that in both series A-C and E-G the same order of efficiency of the host materials remains, it can be suggested that the origin of the efficiency drop of devices based on TADF emitter: F27DMCz layers does not lie in the specific guest-host interactions. The most credible explanation of the suppressing of TADF efficiency is the energy mismatch of the molecular orbitals on the interfaces of layers where radiative recombination takes place. The LUMO level of F27 DMCz makes energetic barrier of $0.56 \mathrm{eV}$ for electrons to transfer from TSPO1 layer to a recombination zone while there is no such a barrier for F36DMCz and MCP (Fig. 6). It can be predicted that improvement of the OLED structure including a selection of the appropriate hole blocking and/or electron transport layers may optimize energy transfer and lead to a considerable enhancement of the efficiency of devices based on F36DMCz. One more possible reason of the best host performance of F36DMCz could be its non-dispersive hole transport with relatively high mobility in contrast to that of other hosts studied (Fig. 3). TADF devices A and phosphorescent device $\mathrm{E}$ based on F36DMCz as the host showed the best maximum EQE values of 8.1 and $12.2 \%$, respectively (Fig. 7, S3). Meanwhile, FTMCz exhibited the worst performance in TADF devices $\mathrm{C}$ and $\mathrm{G}$ based on DAcIPN and DACT-II, showing 4 and $5.6 \mathrm{~lm} / \mathrm{W}$ of maximum power efficiency, 11 and $14.8 \mathrm{~cd} / \mathrm{A}$ of maximum current efficiency, respectively (Fig. 7, S5, S9). Thus, it is expected that carbazolyl moiety substituted by methoxy groups at C- 3 and C- 6 positions has the highest potential for the further developments of bipolar host materials.

Since the first triplet excited state energy values of the studied hosts

Table 4

Structures and electroluminescence parameters of OLEDs.

\begin{tabular}{|c|c|c|c|c|c|c|c|c|}
\hline Device & Host & $\mathrm{L}_{\max }, 10^{3} \mathrm{~cd} / \mathrm{m}^{2 \mathrm{a}}$ & $\eta_{\mathrm{c}}, \mathrm{cd} / \mathrm{A}^{\mathrm{b}}$ & $\eta_{\mathrm{p}}, \operatorname{lm} / \mathrm{W}^{\mathrm{c}}$ & EQE, $\%^{\mathrm{d}}$ & Roll-off Efficiency, \% ${ }^{\mathrm{e}}$ & $\lambda, \mathrm{nm}^{\mathrm{f}}$ & $\mathrm{CIE}^{\mathrm{g}}$ \\
\hline \multicolumn{9}{|c|}{ ITO $/ \mathrm{MoO}_{3}(1 \mathrm{~nm}) / \mathrm{NPB}(45 \mathrm{~nm}) / \mathrm{DAcIPN}: h o s t(10 \mathrm{wt} \%, 30 \mathrm{~nm}) / \mathrm{TSPO} 1(5 \mathrm{~nm}) / \mathrm{TmPyPb}(40 \mathrm{~nm}) / \mathrm{LiF}(0.5 \mathrm{~nm}) / \mathrm{Al}$} \\
\hline A & F36DMCz & 38.7 & 28.9 & 9.2 & 8.1 & 4 & 533 & $(0.32,0.61)$ \\
\hline B & F27DMCz & 23.5 & 16.8 & 6.3 & 5 & 6 & 521 & $(0.27,0.56)$ \\
\hline $\mathrm{C}$ & FTMCz & 12.3 & 11 & 4 & 3.1 & 32 & 538 & $(0.33,0.60)$ \\
\hline \multicolumn{9}{|c|}{ ITO/MoO $3(1 \mathrm{~nm}) / \mathrm{NPB}(45 \mathrm{~nm}) / \mathrm{DACT}-\mathrm{II}: h o s t(10 \mathrm{wt} \%, 30 \mathrm{~nm}) / \mathrm{TSPO} 1(5 \mathrm{~nm}) / \mathrm{TmPyPb}(40 \mathrm{~nm}) / \mathrm{LiF}(0.5 \mathrm{~nm}) / \mathrm{Al}$} \\
\hline E & F36DMCz & 44.5 & 41.6 & 15.1 & 12.2 & 29 & 532 & $(0.31,0.58)$ \\
\hline $\mathrm{F}$ & F27DMCz & 35.9 & 21.4 & 7.6 & 6.7 & 16 & 521 & $(0.27,0.54)$ \\
\hline G & FTMCz & 24.4 & 14.8 & 5.6 & 4.3 & 19 & 534 & $(0.32,0.58)$ \\
\hline \multicolumn{9}{|c|}{ ITO/HAT-CN(4 nm)/NPB(45 nm)/Ir(ppy) $3:$ host $(5 \mathrm{wt} \%, 15 \mathrm{~nm}) / \mathrm{TSPO} 1(5 \mathrm{~nm}) / \mathrm{TPBi}(45 \mathrm{~nm}) / \mathrm{LiF}(0.5 \mathrm{~nm}) / \mathrm{Al}$} \\
\hline $\mathrm{H}$ & F27DMCz & 53.1 & 44.1 & 36.8 & 12.6 & 20 & 509 & - \\
\hline I & FTMCz & 9 & 14 & 11.1 & 4.3 & 30 & 508 & - \\
\hline \multicolumn{9}{|c|}{ ITO/HAT-CN(4 nm)/NPB(45 nm)/Ir(piq) 2 (acac):host(5 wt $\%, 15 \mathrm{~nm}) / \mathrm{TSPO} 1(5 \mathrm{~nm}) / \mathrm{TPBi}(45 \mathrm{~nm}) / \mathrm{LiF}(0.5 \mathrm{~nm}) / \mathrm{Al}$} \\
\hline $\mathrm{J}$ & F36DMCz & 15.7 & 7.6 & 4.8 & 7.3 & 18 & 623 & - \\
\hline $\mathrm{K}$ & F27DMCz & 14.1 & 8.4 & 5.1 & 8.5 & 33 & 623 & - \\
\hline $\mathrm{L}$ & FTMCz & 8.3 & 3.9 & 3.2 & 4.9 & 47 & 629 & - \\
\hline
\end{tabular}

\footnotetext{
a Maximum brightness.

b Maximum current efficiency.

c Maximum power efficiency.

d Maximum external quantum efficiency.

e Calculated at brightness of $5000 \mathrm{~cd} \mathrm{~m}^{-2}$.

${ }^{\mathrm{f}}$ Wavelength of the EL spectrum peak at $6 \mathrm{~V}$.

CIE 1931 UCS coordinates at $10 \mathrm{~V}$.
} 


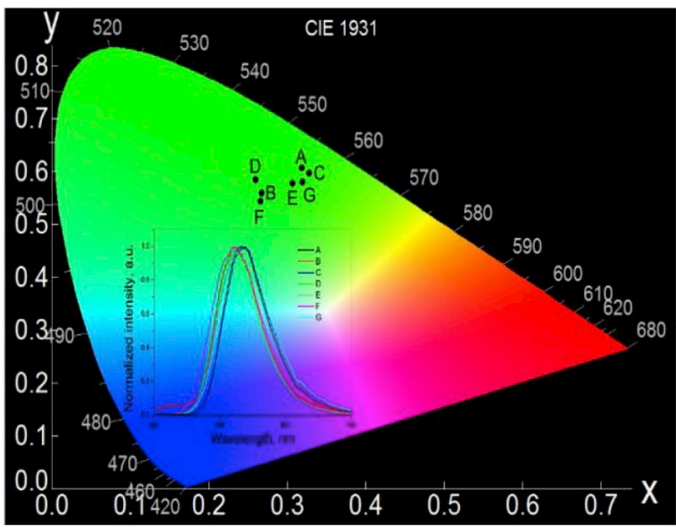

(a)

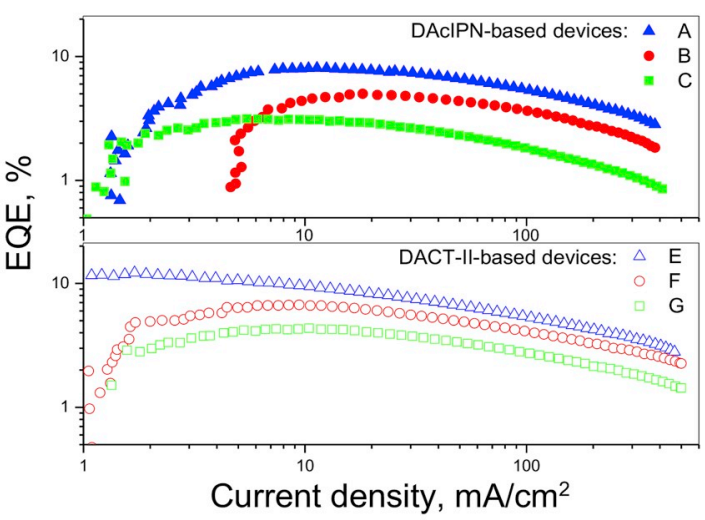

(b)

Fig. 7. (a) CIE 1931 UCS diagram for the EL spectra of the fabricated OLEDs recorded at $8 \mathrm{~V}$ and EQE characteristics (b). Inset: EL spectra of TADF OLEDs recorded at $10 \mathrm{~V}$.

(Table 3) are much higher than triplet energy levels of dopants $\operatorname{Ir}(\mathrm{ppy})_{3}$ (2.4 eV [33]) and $\operatorname{Ir}(\mathrm{piq})_{2}$ (acac) (1.97 eV [34]), efficient triplet energy transfer from host to guest was expected. Consequently, the EL spectra PhOLEDs are typical for an emission attributed to emitters (Figs. S10-14). However, there are slight spectral bands related to the host emission for devices $\mathrm{H}$ and I. Nevertheless, the low turn-on voltages of $\sim 3 \mathrm{~V}$ for these PhOLEDs are the manifestation of an excellent charge transport from electrodes to the light-emitting layers. Despite the similarity of energy levels of TADF OLEDs and PhOLEDs (Fig. 6), high triplet energy of F27DMCz helps to employ triplet excitons more successfully in the system phosphorescent emitter: F27DMCz rather than in the system TADF emitter: F27DMCz. The explanation of this observation may lie in the better energetic match of frontier orbitals of F27DMCz and phosphorescent emitters with subsequent efficient energy transfer. As the result, the highest values of EQE of fabricated PhOLEDs were observed F27DMCz-based devices (12.6\% for device $\mathrm{H}$ and $8.5 \%$ for device $\mathrm{K}$ ). With EQE values of phosphorescent devices lower than 5\%, FTMCz made worse performance. On the basis of the present study, F36DMCz and F27DMCz can be regarded as competitive hosts for doped emitting layers of OLEDs with a TADF or phosphorescent guests $[5,6]$.

To additionally estimate hosting properties of compounds F36DMCz, F27DMCz and FTMCz, roll-off efficiencies of the fabricated devices were analysed. Decreases (in \%) of EQEs of the fabricated devices relative to their maximum EQEs are summarized in Table 4. It should be noted that maximum EQEs were obtained for the most of devices at relatively high brightness apparently because of charge balance achieved at high electric fields (Fig. 7 b). This observation is in agreement with the results of time-of-flight measurements which showed slight differences in hole and electron motilities and electric field dependences (Fig. 3). The lowest roll-off efficiency of $4 \%$ was observed for device A. This observation can be attributed to either higher charge mobilities or more balanced hole and electron transport for the host F36DMCz in comparison to those of F27DMCz and FTMCz (Fig. 3). Since different emitters (DAcIPN, DACT-II, $\operatorname{Ir}(\mathrm{ppy})_{3}$, or $\operatorname{Ir}(\mathrm{pi}-$ q) $)_{2}$ (acac)) were used to test hosting properties of compounds F36DMCz, F27DMCz and FTMCz, different roll-off efficiencies of the devices were observed. These differences may be related to such highenergetic processes as singlet-polaron and triplet-polaron quenches, singlet-singlet, singlet-triplet, and triplet-triplet annihilations the effects of which increase with the increase of applied electric fields [35,36]. The lowest roll-off efficiencies were observed for devices based on F36DMCz as a host (Table 4).

\section{Conclusions}

Derivatives of di(tetra)methoxycarbazole and dibenzofuran with donor-acceptor-donor structure were synthesized as potential host materials for green and red phosphorescent and TADF OLEDs For the estimation of structure-property relationship, thermal, photophysical, photoelectrical, charge transporting and electrochemical properties of the synthesized compounds were studied. Modification of electron-donating carbazole unit with methoxy groups allowed to obtain compounds with higher electrochemical and thermal stability, higher glass transition temperatures and more balanced charge transporting properties compared with the compounds containing non-substituted carbazole. The synthesized compounds showed triplet energies of $2.86-2.96 \mathrm{eV}$. They were tested as the hosts in phosphorescent and TADF-based OLEDs, which showed maximum external quantum efficiency exceeding $12 \%$.

\section{Acknowledgements}

This research was funded by European Social Fund (Project No 09.3.3-LMT-K-712-02-0105).

DG acknowledges to the ERDF PostDoc grant No. 1.1.1.2/VIAA/1/ $16 / 177$.

\section{Appendix A. Supplementary data}

Supplementary data to this article can be found online at https:// doi.org/10.1016/j.dyepig.2019.107781.

\section{References}

[1] Bučinskas A, Volyniuk D, Danyliv Y, Grazulevicius JV, Baryshnikov G, Minaev B, et al. N-annelated perylenes as effective green emitters for OLEDs. RSC Adv 2015:5:78150-9.

[2] Chen Y, Yang L, Huang Y, Obolda A, Abdurahman A, Lu Z, et al. Highly efficient fluorescent organic light-emitting devices using a luminescent radical as the sensitizer. J Phys Chem Lett 2019;10:48-51.

[3] Kirlikovali KO, Spokoyny AM. The long-lasting blues: a new record for phosphorescent organic light-emitting diodes. Chem 2017;3:385-7.

[4] Li B, Li Z, Hu T, Zhang Y, Wang Y, Yi Y, et al. Highly efficient blue organic lightemitting diodes from pyrimidine-based thermally activated delayed fluorescence emitters. J Mater Chem C 2018;6:2351-9.

[5] Tao Y, Yang C, Qin J. Organic host materials for phosphorescent organic lightemitting diodes. Chem Soc Rev 2011;40:2943-70.

[6] Dong Q, Lian H, Gao Z, Guo Z, Xiang N, Zhong Z, et al. Novel spirofluorene/indole/ carbazole-based hole transport materials with high triplet energy for efficient green 
phosphorescent organic light-emitting diodes. Dyes Pigments 2017;137:84-90.

[7] Dong Q, Tai F, Lian H, Chen Z, Hu M, Huang J, et al. Thermally stable bipolar host materials for high efficiency phosphorescent green and blue organic light-emitting diodes. Dyes Pigments 2017;143:470-8.

[8] Jia B, Lian H, Sun T, Guo H, Cheng X, Wu J, et al. Efficient green phosphorescent organic light-emitting diodes enabled with new and thermally stable carbazole/ pyridine derivatives as hosts. Dyes Pigments 2018;159:298-305.

[9] Jia B, Lian H, Sun T, Wei J, Yang J, Zhou H, et al. New bipolar host materials based on methyl substituted pyridazine for high-performance green and red phosphorescent OLEDs. Dyes Pigments 2019;168:212-8.

[10] Fukagawa H, Shimizu T, Kamada T, Yui S, Hasegawa M, Morii K, et al. Highly efficient and stable organic light-emitting diodes with a greatly reduced amount of phosphorescent emitter. Sci Rep 2015;5:9855.

[11] Chatterjee T, Wong K-T. Perspective on host materials for thermally activated delayed fluorescence organic light emitting diodes. Adv Opt Mater 2019;7:1800565.

[12] Jhulki S, Moorthy JN. Small molecular hole-transporting materials (HTMs) in organic light-emitting diodes (OLEDs): structural diversity and classification. J Mater Chem C 2018;6:8280-325.

[13] Wex B, Kaafarani BR. Perspective on carbazole-based organic compounds as emitters and hosts in TADF applications. J Mater Chem C 2017;5:8622-53.

[14] Zhang S, Chen R, Yin J, Liu F, Jiang H, Shi N, et al. Tuning the optoelectronic properties of 4,4'- N, N '-Dicarbazole-biphenyl through heteroatom linkage: new host materials for phosphorescent organic light-emitting diodes. Org Lett 2010;12:3438-41.

[15] Bucinskas A, Bagdziunas G, Tomkeviciene A, Volynyuk D, Kostiv N, Gudeika D, et al. Structure-property relationship of isomeric diphenylethenyl-disubstituted dimethoxycarbazoles. RSC Adv 2015;5:49577-89.

[16] Tomkeviciene A, Grazulevicius JV, Kazlauskas K, Gruodis A, Jursenas S, Ke T-H, et al. Impact of linking topology on the properties of carbazole trimers and dimers. J Phys Chem C 2011;115:4887-97.

[17] Keruckas J, Lygaitis R, Simokaitiene J, Grazulevicius JV, Jankauskas V, Sini G. Influence of methoxy groups on the properties of 1,1-bis(4-aminophenyl)cyclohexane based arylamines: experimental and theoretical approach. J Mater Chem 2012;22:3015-27.

[18] Li W, Li J, Wang F, Gao Z, Zhang S. Universal host materials for high-efficiency phosphorescent and delayed-fluorescence OLEDs. ACS Appl Mater Interfaces 2015;7:26206-16.

[19] Pan J-H, Chou Y-M, Chiu H-L, Wang B-C. Theoretical investigation of organic amines as hole transporting materials: correlation to the Hammett parameter of the substituent, ionization potential, and reorganization energy level. Aust J Chem 2009;62:483-92.

[20] Sakalyte A, Simokaitiene J, Tomkeviciene A, Keruckas J, Buika G, Grazulevicius JV, et al. Effect of methoxy substituents on the properties of the derivatives of carbazole and diphenylamine. J Phys Chem C 2011;115:4856-62.

[21] Sallenave X, Bucinskas A, Salman S, Volyniuk D, Bezvikonnyi O, Mimaite V, et al.
Sensitivity of redox and optical properties of electroactive carbazole derivatives to the molecular architecture and methoxy substitutions. J Phys Chem C 2018;122:10138-52.

[22] Ambrose JF, Nelson RF. Anodic oxidation pathways of carbazoles. J Electrochem Soc 1968;115:1159-64.

[23] D'Andrade BW, Datta S, Forrest SR, Djurovich P, Polikarpov E, Thompson ME Relationship between the ionization and oxidation potentials of molecular organic semiconductors. Org Electron 2005;6:11-20.

[24] Wu F-I, Shih P-I, Yuan M-C, Dixit AK, Shu C-F, Chung Z-M, et al. Novel distyrylcarbazole derivatives as hole-transporting blue emitters for electroluminescent devices. J Mater Chem 2005;15:4753-60.

[25] Li W, Pan Y, Yao L, Liu H, Zhang S, Wang C, et al. A hybridized local and chargetransfer excited state for highly efficient fluorescent OLEDs: molecular design, spectral character, and full exciton utilization. Adv Opt Mater 2014;2:892-901.

[26] Hladka I, Lytvyn R, Volyniuk D, Gudeika D, Grazulevicius JV. W-shaped bipolar derivatives of carbazole and oxadiazole with high triplet energies for electroluminescent devices. Dyes Pigments 2018;149:812-21.

[27] Kukhta NA, da Silva Filho DA, Volyniuk D, Grazulevicius JV, Sini G. Can fluorenone-based compounds emit in the blue region? Impact of the conjugation length and the ground-state aggregation. Chem Mater 2017;29:1695-707.

[28] Skuodis E, Bezvikonnyi O, Tomkeviciene A, Volyniuk D, Mimaite V, Lazauskas A, et al. Aggregation, thermal annealing, and hosting effects on performances of an acridan-based TADF emitter. Org Electron 2018;63:29-40.

[29] Kaji H, Suzuki H, Fukushima T, Shizu K, Suzuki K, Kubo S, et al. Purely organic electroluminescent material realizing $100 \%$ conversion from electricity to light. Nat Commun 2015;6:8476.

[30] Yersin H. Highly efficient OLEDs with phosphorescent materials. Weinheim: WIiley; 2008.

[31] Kim K-H, Moon C-K, Lee J-H, Kim S-Y, Kim J-J. Highly efficient organic light emitting diodes with phosphorescent emitters having high quantum yield and horizontal orientation of transition dipole moments. Adv Mater 2014;26:3844-77.

[32] Song J, Kim K-H, Kim E, Moon C-K, Kim Y-H, Kim J-J, et al. Lensfree OLEDs with over 50\% external quantum efficiency via external scattering and horizontally oriented emitters. Nat Commun 2018;9:3207.

[33] Baldo MA, Forrest SR. Transient analysis of organic electrophosphorescence: I transient analysis of triplet energy transfer. Phys Rev B 2000;62:10958-66.

[34] Bezvikonnyi O, Gudeika D, Volyniuk D, Grazulevicius JV, Bagdziunas G. Pyreny substituted 1,8-naphthalimide as a new material for weak efficiency-roll-off red OLEDs: a theoretical and experimental study. New J Chem 2018;42:12492-502.

[35] Murawski C, Leo K, Gather MC. Efficiency roll-off in organic light-emitting diodes. Adv Mater 2013;25:6801-27.

[36] Bagdžiūnas G, Grybauskaitè G, Kostiv N, Ivaniuk K, Volyniuk D, Lazauskas A. Green and red phosphorescent organic light-emitting diodes with ambipolar hosts based on phenothiazine and carbazole moieties: photoelectrical properties, morphology and efficiency. RSC Adv 2016;6:61544-54. Institute of Solid State Physics, University of Latvia as the Center of Excellence has received funding from
the European Union's Horizon 2020 Framework Programme H2020-WIDESPREAD-01-2016-2017-
TeamingPhase2 under grant agreement No. 739508, project CAMART ${ }^{2}$ 